\title{
ON HYPERSINGULAR INTEGRALS AND LEBESGUE SPACES OF DIFFERENTIABLE FUNCTIONS
}

\author{
BY \\ RICHARD L. WHEEDEN( ${ }^{(1)}$
}

Introduction. If $f(x) \in L^{p}\left(E^{n}\right), 1 \leqq p \leqq \infty, n \geqq 2$, and $\alpha>0$, define $J^{\alpha} f$ by

$$
\left(J^{\alpha} f\right)^{\wedge}(x)=\left(1+|x|^{2}\right)^{-\alpha / 2} \hat{f}(x)
$$

where ${ }^{\wedge}$ denotes the Fourier transformation in the sense of tempered distributions, the Fourier transform of an integrable function $f$ being defined

$$
\hat{f}(x)=(2 \pi)^{-n} \int_{E^{n}} f(z) \exp [-i(x \cdot z)] d z
$$

$J^{\alpha} f$ is called the Bessel potential of order $\alpha$ of $f$, and it is well known that $J^{\alpha} f=f * G_{\alpha}$ where $G_{\alpha} \geqq 0$ and $\int_{E^{n}} G_{\alpha}(x) d x=1$. We denote by $L_{\alpha}^{p}$ all $L^{p}$ functions $f=J^{\alpha} \phi=\phi * G_{\alpha}$ where $\phi \in L^{p}$, and write $\phi=J^{-\alpha} f,\|f\|_{p, \alpha}=\|\phi\|_{p}$. For a discussion of the properties of the $L_{\alpha}^{p}$ spaces, we refer the reader to [1] and [2].

The purpose of this paper is to generalize the following theorem of E. M. Stein stated in [7].

THEOREM. Let $0<\alpha<2,1<p<\infty$ and

$$
f_{\delta}(x)=(2 \pi)^{-n} \int_{|z|>\varepsilon} \frac{f(x+z)-f(x)}{|z|^{n+\alpha}} d z .
$$

Then $f \in L_{\alpha}^{p}$ if and only if $f \in L^{p}$ and $\tilde{f}_{\varepsilon}$ converges in $L^{p}$ norm as $\varepsilon \rightarrow 0$. Moreover, if $\tilde{f}$ is the limit of $\tilde{f}_{\varepsilon}$ then

$$
A_{p, \alpha}\|f\|_{p, \alpha} \leqq\|f\|_{p}+\|f\|_{p} \leqq B_{p, \alpha}\|f\|_{p, \alpha} .
$$

Although it was not stated in [7], Stein was well aware that the theorem remains valid for $p=1$, and that for $p=\infty$ the necessary and sufficient condition that $f \in L_{\alpha}^{\infty}$ is that $f \in L^{\infty}$ and $\left\|\tilde{f}_{\varepsilon}\right\|_{\infty}$ be uniformly bounded.

Stein's theorem may be generalized as follows. Let $0<\alpha<2$ and

$$
\tilde{f}_{\varepsilon}(x)=(2 \pi)^{-n \cdot} \int_{|z|>\varepsilon}[f(x-z)-f(x)] \frac{\Omega\left(z^{\prime}\right)}{|z|^{n+\alpha}} d z
$$

where $z^{\prime}=z /|z|$ for $|z| \neq 0$ and $\Omega$ is a real-valued function which is homogeneous of degree zero and infinitely differentiable $\left({ }^{2}\right)$ on $\sum=\{z:|z|=1\}$. In addition, $\Omega$ satisfies

Received by the editors May 5, 1967 and, in revised form, July 17, 1967.

(1) Supported by National Science Foundation postdoctoral fellowship.

${ }^{(2)}$ See Remark 2 at the end of $\$ 1$. 
$\int_{\Sigma} z_{j}^{\prime} \Omega\left(z^{\prime}\right) d z^{\prime}=0$ for each $j=1, \ldots, n, z=\left(z_{1}, \ldots, z_{n}\right)$, when $1 \leqq \alpha<2$. Then

THEOREM 1. If $f \in L_{\alpha}^{p}, 0<\alpha<2,1<p<\infty$, then $\tilde{f}_{\varepsilon}$ converges in $L^{p}$ to a function $\tilde{f}$ with $\|\tilde{f}\|_{p} \leqq c_{p, \alpha, \Omega}\|f\|_{p, \alpha}$. If $\Omega=1$, then the result is true for $p=1$ and its analogue for $p=\infty$ is that $\left\|\tilde{f_{\varepsilon}}\right\|_{\infty}$ is uniformly bounded.

Conversely, if $f \in L^{p}, 1<p<\infty$, and each

$$
(2 \pi)^{-n} \int_{|z|>\varepsilon}[f(x-z)-f(x)] \frac{\Omega_{i}(z)}{|z|^{n+\alpha}} d z
$$

converges in $L^{p}$ to limit $\tilde{f_{i}}$ where $\left\{\Omega_{i}\right\}$ is a normalized basis for the spherical harmonics of some fixed degree $m, m \neq 1$ when $1 \leqq \alpha<2$, then $f \in L_{\alpha}^{p}$ and $\|f\|_{p, \alpha} \leqq$ $c_{p, \alpha, m}\left(\sum_{i}\left\|\tilde{f}_{i}\right\|_{p}+\|f\|_{p}\right)$. If $m=0$ the result is valid for $p=1$ and its analogue for $p=\infty$ is that $f \in L_{\alpha}^{\infty}$ if $f \in L^{\infty}$ and $\left\|f_{\varepsilon}\right\|_{\infty}$ is uniformly bounded.

Before proceeding, we note that when $\alpha=1$ and $1<p<\infty$, the first part of Theorem 1 is a rather simple corollary of the fact that singular integrals preserve $L^{p}$ spaces. In fact, approximating $L_{1}^{p}$ by the class $C_{0}^{\infty}$ of infinitely differentiable functions with compact support and integrating by parts (see [11, Lemma 1 of §2]), it is not hard to see that $\tilde{f}_{\varepsilon}(x)$ converges in $L^{p}$ to

$$
-\sum_{j=1}^{n} \text { p.v. }(2 \pi)^{-n} \int_{E^{n}} f_{j}(x-z) \frac{\Omega_{j}\left(z^{\prime}\right)}{|z|^{n}} d z
$$

where $\Omega_{j}\left(z^{\prime}\right)=z_{j}^{\prime} \Omega\left(z^{\prime}\right), f_{j}(x)=\left(\partial f / \partial x_{j}\right)(x)$.

Although we will restrict $\alpha$ to the range $0<\alpha<2$, Theorem 1 has analogues for larger $\alpha$. If $k \geqq 1$ is a fixed integer, let $f \in L_{k-1}^{p}, 1<p<\infty$. If $\beta=\left(\beta_{1}, \ldots, \beta_{n}\right)$ where the $\beta_{j}$ are nonnegative integers and $|\beta|=\beta_{1}+\cdots+\beta_{n}, \beta !=\beta_{1} ! \cdots \beta_{n} !, z^{\beta}=z_{1}^{\beta_{1}} \cdots z_{n}^{\beta_{n}}$ then for $|\beta| \leqq k-1$, let $f_{\beta}(x)$ denote the $L^{p}$ function which is the derivative of $f$ of order $\beta$. For $k-1<\alpha<k+1$, consider the truncated hypersingular integral

$$
\tilde{f_{\varepsilon}}(x)=(2 \pi)^{-n} \int_{|z|>\varepsilon}\left[f(x+z)-\sum_{|\beta| \leq k-1} \frac{f_{\beta}(x)}{\beta !} z^{\beta}\right] \frac{\Omega\left(-z^{\prime}\right)}{|z|^{n+\alpha}} d z
$$

where $\Omega$ is a real-valued function which is homogeneous of degree zero and infinitely differentiable on $\sum$. In addition, $\Omega$ satisfies $\int_{\Sigma} z^{\prime \beta} \Omega\left(z^{\prime}\right) d z^{\prime}=0$ for all $|\beta|=k$ when $k \leqq \alpha<k+1$. Then

THEOREM 2. If $f \in L_{\alpha}^{p}, 1<p<\infty, k-1<\alpha<k+1$, then $f_{\varepsilon}$ converges in $L^{p}$ to a function $\tilde{f}$ with $\|\tilde{f}\|_{p} \leqq c_{p, \alpha, s}\|f\|_{p, \alpha}$.

Conversely, if $f \in L_{k-1}^{p}, 1<p<\infty$, and each

$$
(2 \pi)^{-n} \int_{|z|>\varepsilon}\left[f(x+z)-\sum_{|\beta| \leq k-1} \frac{f_{\beta}(x)}{\beta !} z^{\beta}\right] \frac{\Omega_{i}\left(z^{\prime}\right)}{|z|^{n+\alpha}} d z
$$

converges in $L^{p}$ to $\tilde{f}_{i}$ where $\left\{\Omega_{i}\right\}$ is a normalized basis for the spherical harmonics of some fixed degree $m, m \neq k, k-2, \ldots$ when $k \leqq \alpha<k+1$, then $f \in L_{\alpha}^{p}$ and

$$
\|f\|_{p, \alpha} \leqq c_{p, \alpha, m}\left(\sum_{i}\left\|\tilde{f}_{i}\right\|_{p}+\|f\|_{p}\right)
$$


Theorem 2 also has versions for $p=1$ and $p=\infty$, but they depend on $k$ and $\alpha$ and we shall not give them explicitly.

In proving Theorem 1, we will take for granted the basic facts from the theory of singular integrals (see [4]). In addition, we use several formulas for spherical harmonics. In particular, if $\Omega\left(z^{\prime}\right)$ is sufficiently smooth and has mean-value zero on $\sum$, and its expansion in spherical harmonics is $\sum_{1}^{\infty} Y_{m}\left(z^{\prime}\right)$, then the principal-valued Fourier transform $\hat{K}(z)$ of $K(z)=|z|^{-n} \Omega\left(z^{\prime}\right)$ is given by

$$
R(z)=\sum_{1}^{\infty} \gamma_{m} Y_{m}\left(z^{\prime}\right), \quad \gamma_{m}=(-i)^{m} \Gamma\left(\frac{m}{2}\right) / 2^{n} \pi^{n / 2} \Gamma\left(\frac{m+n}{2}\right),
$$

where

$$
\hat{K}(z)=\lim _{\varepsilon \rightarrow 0 ; \omega \rightarrow \infty}(2 \pi)^{-n} \int_{\varepsilon<|y|<\omega} K(y) \exp [-i(y \cdot z)] d y .
$$

Moreover, if $\left\{\Omega_{i}\right\}$ is a normalized basis for the spherical harmonics of a fixed degree $m \geqq 1$ then $\sum_{i} \Omega_{i}^{2}(x)$ is a constant depending on $m$ [6, p. 243(2)]. An immediate corollary is that if $f \in L^{p}, 1<p<\infty$, and $R_{i} f=$ p.v. $(2 \pi)^{-n} f *\left(\Omega_{i}(x) /|x|^{n}\right)$ then $f=c_{m} \sum R_{i}^{2} f$ almost everywhere and, in particular, $\|f\|_{p} \leqq c_{m, p} \sum\left\|R_{i} f\right\|_{p}$.

Finally, the following formula will be used repeatedly (see [6, p. 247 and p. 178]):

$$
\int_{\Sigma} Y_{m}\left(z^{\prime}\right) \exp \left[-i s\left(x^{\prime} \cdot z^{\prime}\right)\right] d z^{\prime}=i^{m}(2 \pi)^{\gamma} \frac{J_{m+\gamma}(s)}{s^{\gamma}} Y_{m}\left(-x^{\prime}\right)
$$

where $x^{\prime}$ is a unit vector, $Y_{m}$ is a spherical harmonic of degree $m \geqq 0, \gamma=(n-2) / 2$, and $J_{v}$ is the Bessel function of order $\nu$.

The following lemma, stated in [7], will also be essential.

LEMMA (0.1). If $\alpha>0$ then

$$
|x|^{\alpha}=\left(1+|x|^{2}\right)^{\alpha / 2} d \hat{\mu}, \quad\left(1+|x|^{2}\right)^{\alpha / 2}=|x|^{\alpha} d \hat{\sigma}+d \hat{\tau}
$$

where $d \hat{\mu}, d \hat{\sigma}, d \hat{\tau}$ denote the Fourier transforms of finite measures $d \mu, d \sigma, d \tau$.

We will prove the sufficiency of Theorem 1 in $\$ 1$ and the necessity in $\$ 2$. We also list several remarks of some independent interest at the ends of both sections.

1. In this section, we prove the sufficiency of Theorem 1 . Thus let

$$
f_{\varepsilon}(x)=(2 \pi)^{-n} \int_{|z|>\varepsilon}[f(x-z)-f(x)] \frac{\Omega\left(z^{\prime}\right)}{|z|^{n+\alpha}} d z
$$

for $0<\alpha<2$, where $\Omega$ is homogeneous of degree zero, infinitely differentiable on $|z|=1$, and orthogonal on $|z|=1$ to each $z_{j}, j=1, \ldots, n$, when $1 \leqq \alpha<2$. Hence $\Omega\left(z^{\prime}\right)=\sum_{0}^{\infty} Y_{m}\left(z^{\prime}\right)$ and $Y_{1} \equiv 0$ when $1 \leqq \alpha<2$.

We will suppose for the time being that $f \in C_{0}^{\infty}$ and show that for $1<p<\infty$

(a) $f_{\varepsilon}$ converges in $L^{p}$ as $\varepsilon \rightarrow 0$, and

(b) $\left\|f_{\varepsilon}\right\|_{p} \leqq c_{p, \alpha, \Omega}\|f\|_{p, \alpha}$. 
To prove this, we will essentially compare $\tilde{f}_{\varepsilon}$ with the sum of the Poisson integral of $J^{-\alpha} f$ and the Poisson integral of a singular integral of $J^{-\alpha} f$. It is the presence of the singular integral which generally prevents (a) and (b) from holding when $p=1$ and $p=\infty$, and we will handle these cases later. The singular integral will be generated by a kernel

$$
|z|^{-n} \sum_{1}^{\infty} d_{m}^{(\alpha)} Y_{m}\left(z^{\prime}\right)
$$

and we begin with a discussion leading to the determination of the $d_{m}^{(\alpha)}$.

For $f \in C_{0}^{\infty}$, write $\tilde{f}_{\varepsilon}=\tilde{A}_{\varepsilon}+\widetilde{B}_{\varepsilon}$ where

$$
\tilde{A}_{\varepsilon}(x)=(2 \pi)^{-n} \int_{|z|>\varepsilon}[f(x-z)-f(x)] \frac{Y_{0}\left(z^{\prime}\right)}{|z|^{n+\alpha}} d z
$$

and

$$
\widetilde{B}_{\varepsilon}(x)=(2 \pi)^{-n} \int_{|z|>\varepsilon} f(x-z) \frac{\sum_{1}^{\infty} Y_{m}\left(z^{\prime}\right)}{|z|^{n+\alpha}} d z=(2 \pi)^{-n} \sum_{1}^{\infty} \int_{|z|>\varepsilon} f(x-z) \frac{Y_{m}\left(z^{\prime}\right)}{|z|^{n+\alpha}} d z .
$$

We will compute $\tilde{f}_{\varepsilon}^{\hat{\varepsilon}}=\tilde{A}_{\varepsilon}^{\hat{\varepsilon}}+\tilde{B}_{\varepsilon}^{\wedge}$ and $\lim _{\varepsilon \rightarrow 0} \tilde{f_{\varepsilon}^{\hat{\varepsilon}}}$.

$$
\tilde{A}_{\varepsilon}^{\wedge}(x)=(2 \pi)^{-n} \hat{f}(x) \int_{|z|>\varepsilon} Y_{0}\left(z^{\prime}\right)[\exp [-i(x \cdot z)]-1] \frac{d z}{|z|^{n+\alpha}} .
$$

Using polar coordinates $z=(s /|x|) z^{\prime}, \varepsilon|x| \leqq s<\infty,\left|z^{\prime}\right|=1$, and applying (0.1) we obtain

$$
\tilde{A_{\varepsilon}^{\wedge}}(x)=(2 \pi)^{-n / 2} \hat{f}(x)|x|^{\alpha} Y_{0}\left(-x^{\prime}\right) w_{0}(\varepsilon|x|),
$$

where for $r>0, \gamma=(n-2) / 2$,

$$
w_{0}(r)=w_{0}^{(\alpha)}(r)=\int_{r}^{\infty} s^{-\alpha-1}\left[\frac{J_{\gamma}(s)}{s^{\gamma}}-\frac{1}{2^{\gamma} \Gamma(\gamma+1)}\right] d s .
$$

Now

$$
\tilde{B}_{\varepsilon}^{\wedge}(x)=(2 \pi)^{-n} \hat{f}(x) \sum_{1}^{\infty} \int_{|z|>\varepsilon} Y_{m}\left(z^{\prime}\right) \exp [-i(x \cdot z)] \frac{d z}{|z|^{n+\alpha}},
$$

and in the same way

$$
\tilde{B}_{\varepsilon}(x)=(2 \pi)^{-n / 2} \hat{f}(x)|x|^{\alpha} \sum_{1}^{\infty} i^{m} Y_{m}\left(-x^{\prime}\right) w_{m}(\varepsilon|x|),
$$

where for $r>0, \gamma=(n-2) / 2$,

$$
w_{m}(r)=w_{m}^{(\alpha)}(r)=\int_{r}^{\infty} s^{-\alpha-\gamma-1} J_{m+\gamma}(s) d s .
$$

If $0<\alpha<1$ and $m \geqq 1$, it follows from [10, p. $391(1)]$, that $\lim _{r \rightarrow 0} w_{m}(r)=$ $\int_{0}^{\infty} s^{-\alpha-\gamma-1} J_{m+\gamma}(s) d s$ exists and equals $2^{-\alpha-\gamma-1} \Gamma((m-\alpha) / 2) / \Gamma((m+n+\alpha) / 2)$. If on 
the other hand $1 \leqq \alpha<2$ then $Y_{1} \equiv 0$ and $\lim _{r \rightarrow 0} w_{m}(r)$ again exists and has the same value provided $m \geqq 2$.

Writing $s^{-\alpha-1}=-\alpha^{-1}(d / d s)\left(s^{-\alpha}\right)$ and integrating by parts,

$$
w_{0}(r)=-\alpha^{-1}\left\{\left.s^{-\alpha}\left(\frac{J_{y}(s)}{s^{\gamma}}-\frac{1}{2^{\gamma} \Gamma(\gamma+1)}\right)\right|_{r} ^{\infty}+\int_{r}^{\infty} s^{-\gamma-\alpha} J_{\gamma+1}(s) d s\right\}
$$

by $[10$, p. 45$]$. The integrated term is $O\left(s^{-\alpha}\right)$ and so tends to zero as $s \rightarrow \infty$. On the other hand, by $[10$, p. $40(8)]$, the integrated term equals

$$
s^{-\alpha-\gamma} \sum_{1}^{\infty} \frac{(-1)^{m}(s / 2)^{\gamma+2 m}}{m ! \Gamma(\gamma+m+1)}=O\left(s^{-\alpha-\gamma} s^{\gamma+2}\right)=O\left(s^{2-\alpha}\right)
$$

for small s. Since $2-\alpha>0$, it follows from $[10$, p. $391(1)]\left({ }^{3}\right)$, that $\lim _{r \rightarrow 0} w_{0}(r)$ $=-\alpha^{-1} \int_{0}^{\infty} s^{-\gamma-\alpha} J_{\gamma+1}(s) d s$ exists and equals

$$
-\alpha^{-1} 2^{-\alpha-\gamma} \Gamma\left(\frac{2-\alpha}{2}\right) / \Gamma\left(\frac{n+\alpha}{2}\right)=2^{-\alpha-\gamma-1} \Gamma\left(-\frac{\alpha}{2}\right) / \Gamma\left(\frac{n+\alpha}{2}\right) .
$$

Let

$$
w_{m}^{(\alpha)}=\lim _{r \rightarrow 0} w_{m}^{(\alpha)}(r)=2^{-\alpha-\gamma-1} \Gamma\left(\frac{m-\alpha}{2}\right) / \Gamma\left(\frac{m+n+\alpha}{2}\right) \text { and } c_{m}^{(\alpha)}=(2 \pi)^{-n / 2}(-i)^{m} w_{m}^{(\alpha)}
$$

and recall that when $1 \leqq \alpha<2, w_{1}^{(\alpha)}$ and $c_{1}^{(\alpha)}$ are not defined and $Y_{1} \equiv 0$. If $S\left(z^{\prime}\right)$ $=\sum_{0}^{\infty} c_{m}^{(\alpha)} Y_{m}\left(z^{\prime}\right)\left(c_{m}^{(\alpha)}=O\left(m^{-n / 2-\alpha}\right)\right.$ by $[5$, p. $\left.47(5)]\right)$, we set

$$
f(x, \varepsilon)=\int_{E^{n}} f(x+z)\left[|z|^{\alpha} S\left(z^{\prime}\right) \exp [-\varepsilon|z|]\right]^{\wedge} d z .
$$

LeMma (1.1). If $f \in C_{0}^{\infty}$ and $1<p<\infty$ then

(a') $f_{\varepsilon}(x)-\tilde{f}(x, \varepsilon)$ converges in $L^{p}$ as $\varepsilon \rightarrow 0$ and

(b') $\left\|f_{\varepsilon}^{\prime}(\cdot)-f^{f}(\cdot, \varepsilon)\right\|_{p} \leqq c_{p, \alpha, \Omega}\|f\|_{p, \alpha}$.

This lemma will establish (a) and (b), for writing $S\left(z^{\prime}\right)=c+\sum_{1}^{\infty} c_{m}^{(\alpha)} Y_{m}\left(z^{\prime}\right)$ $=c+R\left(z^{\prime}\right), c=c_{0}^{(\alpha)} Y_{0}\left(z^{\prime}\right)$,

$$
\begin{aligned}
f(x, \varepsilon)= & c \int_{E^{n}} \hat{f}(z)|z|^{\alpha} \exp [-i(x \cdot z)-\varepsilon|z|] d z \\
& +\int_{E^{n}} \hat{f}(z)|z|^{\alpha} R\left(z^{\prime}\right) \exp [-i(x \cdot z)-\varepsilon|z|] d z .
\end{aligned}
$$

By Lemma (0.1), $\hat{f}(z)|z|^{\alpha}=\left(J^{-\alpha} f * d \mu\right)^{\wedge}$ and since $R\left(z^{\prime}\right)$ is the principal-valued Fourier transform of

$$
|z|^{-n} \sum_{1}^{\infty} d_{m}^{(\alpha)} Y_{m}\left(z^{\prime}\right), \quad d_{m}^{(\alpha)}=c_{m}^{(\alpha)} \gamma_{m}^{-1},
$$

(3) When $n=2(\gamma=0)$, it is necessary to integrate $w_{0}(r)$ by parts twice before applying this formula. 
it follows that $f(x, \varepsilon)$ is the sum of the Poisson integral of $c\left(J^{-\alpha} f * d \mu\right)$ and the Poisson integral of a singular integral of $J^{-\alpha} f * d \mu$. Since $J^{-\alpha} f \in L^{p}$ and $1<p<\infty$, $f(x, \varepsilon)$ converges in $L^{p}$ as $\varepsilon \rightarrow 0$ and $\|\tilde{f}(x, \varepsilon)\|_{p} \leqq c_{p, \alpha, \Omega}\left\|J^{-\alpha} f\right\|_{p}=c_{p, \alpha, \Omega}\|f\|_{p, \alpha}$.

We begin proving Lemma (1.1) by computing $\left[|z|^{\alpha} S\left(z^{\prime}\right) \exp [-\varepsilon|z|]\right]^{\wedge}$.

$$
\begin{aligned}
{\left[|z|^{\alpha} Y_{m}\left(z^{\prime}\right) \exp [-\varepsilon|z|]\right]^{\wedge} } & =(2 \pi)^{-n} \int_{E^{n}}|y|^{\alpha} Y_{m}\left(y^{\prime}\right) \exp [-\varepsilon|y|-i(z \cdot y)] d y \\
& =|z|^{-n-\alpha}(2 \pi)^{-n / 2} i^{m} \nu_{m}^{(\alpha)}(\varepsilon /|z|) Y_{m}\left(-z^{\prime}\right),
\end{aligned}
$$

where $\nu_{m}^{(\alpha)}(r)=\int_{0}^{\infty} \exp [-r s] s^{\gamma+\alpha+1} J_{m+\gamma}(s) d s, r>0$. We obtain this by changing to polar coordinates $y=(s /|z|) y^{\prime}$ and applying (0.1). In particular,

$$
f(x, \varepsilon)=(2 \pi)^{-n} \int_{E^{n}} f(x+z) K_{\varepsilon}(z) d z,
$$

where

$$
K_{\varepsilon}(z)=\sum_{0}^{\infty} w_{m}^{(\alpha)} \nu_{m}^{(\alpha)}(\varepsilon /|z|) Y_{m}\left(-z^{\prime}\right) /|z|^{n+\alpha}
$$

LEMMA (1.2). $\left|\nu_{m}^{(\alpha)}(r)\right| \leqq c_{n, \alpha} r^{-n-\alpha}$.

For $\left|J_{m+\gamma}(s)\right| \leqq 1$ and $\left|J_{m+\gamma}(s)\right| \leqq s^{m+\gamma}$ imply $\left|J_{m+\gamma}(s)\right| \leqq s^{\gamma}$ so that

$$
\left|\nu_{m}^{(\alpha)}(r)\right| \leqq \int_{0}^{\infty} \exp [-r s] s^{2 \gamma+\alpha+1} d s=r^{-n-\alpha} \int_{0}^{\infty} \exp [-t] t^{2 \gamma+\alpha+1} d t .
$$

For each fixed $\varepsilon>0,\left[|z|^{\alpha} \exp [-\varepsilon|z|]\right]^{\wedge}$ is bounded as the Fourier transform of an integrable function; moreover by [10, p. $385(2)]$, and $[5$, p. $76(9)], \nu_{0}(r)$ is bounded as $r \rightarrow 0$. Hence for fixed $\varepsilon$ and large $|z|$, it follows from (1.1) that $\left[|z|^{\alpha} \exp [-\varepsilon|z|]\right]^{\wedge}$ $=O\left(|z|^{-n-\alpha}\right)$ and so $\left[|z|^{\alpha} \exp [-\varepsilon|z|]\right]^{\wedge} \in L\left(E^{n}\right)$. Thus $\int_{E^{n}}\left[|z|^{\alpha} \exp [-\varepsilon|z|]\right]^{\wedge} d z=0$, or what is the same thing, $\int_{E^{n}} v_{0}^{(\alpha)}(\varepsilon /|z|)|z|^{-n-\alpha} d z=0$. Since $\int_{\Sigma} Y_{m}\left(z^{\prime}\right) d z^{\prime}=0$ for $m \neq 0$, we obtain $\int_{|z|=1} K_{\varepsilon}(z) d z=0$ (that we may integrate $K_{\varepsilon}$ termwise is clear from Lemma (1.2)), and

$$
f(x, \varepsilon)=(2 \pi)^{-n} \int_{E^{n}}[f(x+z)-f(x)] K_{\delta}(z) d z .
$$

LEMMA (1.3). $\left|w_{m}^{(\alpha)} \nu_{m}^{(\alpha)}(r)-1\right| \leqq A\left\{[(m+1) r]^{1 / 2}+[(m+1) r]^{3 / 2}\right\}$ for all $m$ if $0<\alpha<1$, and for all $m \neq 1$ if $1 \leqq \alpha<2$. In any case, $A$ is independent of $r$ and $m$.

Lemma 3 was proved for $\alpha=1$ in [11], and the proof for other $\alpha$ is similar. For $r \geqq \frac{1}{2},\left|w_{m}^{(\alpha)} \nu_{m}^{(\alpha)}(r)-1\right| \leqq A_{n, \alpha}$ by Lemma (1.2), and Lemma (1.3) is established in this case. By [10, p. 385 (2)], and [5, p. 59 (10)], if $m>\alpha+1$,

$$
\begin{aligned}
\nu_{m}^{(\alpha)}(r)= & 2^{-m-1} \Gamma(m+n+\alpha) / \Gamma\left(\frac{m+n+\alpha+1}{2}\right) \Gamma\left(\frac{m-\alpha-1}{2}\right) \\
& \cdot \int_{0}^{1} t^{(m+n+\alpha-1) / 2}(1-t)^{(m-\alpha-3) / 2} \frac{d t}{\left(r^{2}+t\right)^{(m+n+\alpha) / 2}} .
\end{aligned}
$$


Hence for $m>\alpha+1, v_{m}(r) \leqq \nu_{m}(0)=1 / w_{m}^{(\alpha)}$ by [5, p. $9(5)$ and p. $\left.5(15)\right]$. For $m>\alpha+1$ and $0<r<\frac{1}{2}$, it follows exactly as in [11, Lemma 6, §2], that

$$
\begin{aligned}
\left|w_{m}^{(\alpha)} \nu_{m}^{(\alpha)}(r)-1\right| & =w_{m}^{(\alpha)}\left[\nu_{m}^{(\alpha)}(0)-\nu_{m}^{(\alpha)}(r)\right] \\
& =O\left\{[(m+1) r]^{1 / 2}+[(m+1) r]^{3 / 2}\right\} .
\end{aligned}
$$

It remains only to prove the same estimate for $m \leqq \alpha+1$. Hence for $0<\alpha<1$ we must consider the cases $m=0,1$, and for $1 \leqq \alpha<2$ the cases $m=0,2$.

Suppose $0<\alpha<1$ and write

$$
\nu_{1}^{(\alpha)}(r)=\int_{0}^{\infty} \exp [-r s] s^{\alpha-1} \frac{d}{d s}\left(s^{\gamma+2} J_{y+2}(s)\right) d s
$$

by $[10$, p. 45$]$. Performing an integration by parts,

$$
w_{1}^{(\alpha)} \nu_{1}^{(\alpha)}(r)-1=r w_{1}^{(\alpha)} \nu_{2}^{(\alpha)}(r)+\left\{(1-\alpha) w_{1}^{(\alpha)} \int_{0}^{\infty} \exp [-r s] s^{\alpha+\gamma} J_{\gamma+2}(s) d s-1\right\} .
$$

By (1.3), $\left|r w_{1}^{(\alpha)} \nu_{2}^{(\alpha)}(r)\right| \leqq A_{\alpha, n} r \leqq A_{\alpha, n} r^{1 / 2}$. By [10, p. 385 (2)], and [5, p. 59], and an argument practically identical to that given for $m>\alpha+1$, the expression in curly brackets is $O\left(r^{1 / 2}+r^{3 / 2}\right), 0<r<\frac{1}{2}$, and the lemma is proved for $0<\alpha<1$ and $m=1$. For $0<\alpha<1$ and $m=0$, we can use a similar argument after integrating $\nu_{0}^{(\alpha)}(r)$ by parts twice.

The case $1 \leqq \alpha<2, m=0$ and $m=2$ are analogous, and Lemma (1.3) is proved.

To prove Lemma (1.1) we need one more fact.

Lemma (1.4). If $f \in C_{0}^{\infty}$ then

$$
\|f(x+z)-f(x)\|_{p} \leqq c\|f\|_{p, \beta}|z|^{\beta}
$$

if $0<\beta \leqq 1$ and $1<p<\infty$,

$$
\left\|f(x+z)-f(x)-\sum_{j=1}^{n} z_{j} \frac{\partial f}{\partial x_{j}}(x)\right\|_{p} \leqq c\|f\|_{p, \beta}|z|^{\beta}
$$

if $1 \leqq \beta \leqq 2$ and $1<p<\infty$, and

$$
\|f(x+z)+f(x-z)-2 f(x)\|_{p} \leqq c\|f\|_{p, \beta}|z|^{\beta}
$$

if $0<\beta<2$ and $1 \leqq p \leqq \infty$, with $c$ independent of $f$.

When $\beta$ is an integer and $1<p<\infty$, Lemma (1.4) follows from the identification of $L_{\beta}^{p}$ with the classical Sobolev space of functions with partial derivatives up to order $\beta$ in $L^{p}$. For all other values of $\beta$ and $p$, Lemma (1.4) follows easily from writing $f=J^{\beta}\left(J^{-\beta} f\right)=\left(J^{-\beta} f\right) * G_{\beta}$. See [2] for similar statements.

Returning to the proof of Lemma (1.1), let us first suppose that $0<\alpha<1$. By Lemma (1.2) the $L^{p}$ norm of the part of (1.2) extended over $|z|<\varepsilon$ is majorized by a constant times

$$
\int_{|z|<\varepsilon}\|f(x+z)-f(x)\|_{p}(\varepsilon /|z|)^{-n-\alpha}|z|^{-n-\alpha} d z
$$


which by Lemma (1.4) (i) is in turn majorized by

$$
c\|f\|_{p, \varepsilon^{-}} \varepsilon^{-n-\alpha} \int_{|z|<\varepsilon}|z|^{\beta} d z=c^{\prime}\|f\|_{p, \beta} \varepsilon^{\beta-\alpha}
$$

for any $0<\beta \leqq 1$. Choosing first $\beta=\alpha$ and then $\beta=1$, we see (1.4) is majorized by $c\|f\|_{p, \alpha}$ and tends to zero with $\varepsilon$, respectively.

On the other hand, if $1 \leqq \alpha<2$ then $Y_{1} \equiv 0$ and the part of (1.2) extended over $|z|<\varepsilon$ is unchanged if we replace $f(x+z)-f(x)$ in the integrand by $f(x+z)-f(x)$ $-\sum_{j=1}^{n} z_{j}\left(\partial f / \partial x_{j}\right)(x)$. Applying Lemma (1.4) (ii) and arguing as above with $\beta=\alpha$ and $\beta=2$ respectively, we see the $L^{p}$ norm of this part is bounded by $c\|f\|_{p, \alpha}$ and tends to zero with $\varepsilon$.

Hence since

$$
\tilde{f}_{\varepsilon}(x)=(2 \pi)^{-n} \int_{|z|>\varepsilon}[f(x+z)-f(x)] \sum_{0}^{\infty} Y_{m}(-z)|z|^{-n-\alpha} d z,
$$

Lemma (1.1) will follow once we show that the $L^{p}$ norm of

$$
\int_{|z|>\varepsilon}[f(x+z)-f(x)] \sum_{0}^{\infty} Y_{m}\left(-z^{\prime}\right)\left[w_{m}^{(\alpha)} \nu_{m}^{(\alpha)}\left(\frac{\varepsilon}{|z|}\right)-1\right]|z|^{-n-\alpha} d z
$$

is bounded by $c\|f\|_{p, \alpha}$ and tends to zero with $\varepsilon$. If $0<\alpha<1$, Lemmas (1.3) and (1.4) (i) imply the $L^{p}$ norm of (1.5) is majorized by

$$
c\|f\|_{p, \alpha} \int_{|z|>\varepsilon}|z|^{\alpha}(\varepsilon /|z|)^{1 / 2}|z|^{-n-\alpha} d z=c^{\prime}\|f\|_{p, \alpha} .
$$

On the other hand, for fixed $\delta>\varepsilon$ it is majorized by a constant times

$$
\begin{aligned}
\int_{\varepsilon<|z|<\delta}\|f(x+z)-f(x)\|_{p}(\varepsilon /|z|)^{1 / 2}|z|^{-n-\alpha} d z & \|f\|_{p} \int_{|z|>\delta}(\varepsilon /|z|)^{1 / 2}|z|^{-n-\alpha} d z \\
& \leqq c_{1}\|f\|_{p, 1} \varepsilon^{1 / 2} \int_{\delta}^{\delta} \frac{d t}{t^{\alpha+1 / 2}}+c_{2}\|f\|_{p} \varepsilon^{1 / 2},
\end{aligned}
$$

and so tends to zero with $\varepsilon$. If $1 \leqq \alpha<2$, we replace $f(x+z)-f(x)$ by $f(x+z)-f(x)$ $-\sum_{j=1}^{n} z_{j}\left(\partial f / \partial x_{j}\right)(x)$ in the integrand of $(1.5)$ and argue in the same way.

Having established (a) and (b) for $f \in C_{0}^{\infty}$, we now claim they hold for any $f \in L_{\alpha}^{p}$. For if $f \in L_{\alpha}^{p}$, pick $f_{k} \in C_{0}^{\infty}$ such that $f_{k} \rightarrow f$ in $L_{\alpha}^{p}$. Then for each fixed $\varepsilon>0$ $\left(f_{k}\right)_{\varepsilon} \rightarrow \tilde{f}_{\varepsilon}$ in $L^{p}$ by Young's inequality. Since $\left\|\left(f_{k}\right)_{\varepsilon}\right\|_{p} \leqq c\left\|f_{k}\right\|_{p, \alpha}$, we see by making $k \rightarrow \infty$ that (b) is true for any $f \in L_{\alpha}^{p}$. Given $f \in L_{\alpha}^{p}$, pick $g \in C_{0}^{\infty}$ with $\|f-g\|_{p, \alpha}$ arbitrarily small. With $h=f-g$,

$$
\begin{aligned}
\left\|\tilde{f}_{\varepsilon_{1}}-\tilde{f}_{\varepsilon_{2}}\right\|_{p} & \leqq\left\|\tilde{g}_{\varepsilon_{1}}-\tilde{g}_{\varepsilon_{2}}\right\|_{p}+\left\|\tilde{h}_{\varepsilon_{1}}\right\|_{p}+\left\|\tilde{h}_{\varepsilon_{2}}\right\|_{p} \\
& \leqq\left\|\tilde{g}_{\varepsilon_{1}}-\tilde{g}_{\varepsilon_{2}}\right\|_{p}+2\|h\|_{p, \alpha} .
\end{aligned}
$$

It follows that $\tilde{f}_{\varepsilon}$ converges in $L^{p}$ to a limit $\tilde{f}$ and $\left\|\tilde{f}_{p} \leqq c_{p, \alpha, \Omega}\right\| f \|_{p, \alpha}$ for any $f \in L_{\alpha}^{p}$. 
Our result also has versions for the cases $p=1$ and $p=\infty$ provided $\Omega=1$. Consider first $p=1, \Omega=1$ and $f \in C_{0}^{\infty}$. Then

$$
\begin{aligned}
f(x, \varepsilon) & =c_{0}^{(\alpha)} \int_{E^{n}} f(x+z)\left[|z|^{\alpha} \exp [-\varepsilon|z|]\right]^{\wedge} d z \\
& =\frac{c_{0}^{(\alpha)}}{2} \int_{E^{n}}[f(x+z)+f(x-z)-2 f(x)]\left[|z|^{\alpha} \exp [-\varepsilon|z|]\right]^{\wedge} d z
\end{aligned}
$$

is the Poisson integral of $J^{-\alpha} f * d \mu \in L^{1}\left(E^{n}\right)$. Since

$$
f_{\delta}(x)=\frac{(2 \pi)^{-n}}{2} \int_{|z|>\delta}[f(x+z)+f(x-z)-2 f(x)] \frac{d z}{|z|^{n+\alpha}},
$$

the remainder of the proof that $\tilde{f}_{\varepsilon}$ converges in $L^{1}$ and $\left\|f_{\varepsilon}\right\|_{1} \leqq c\|f\|_{1, \alpha}$ for $f \in L_{\alpha}^{1}$ follows the lines of the argument for $1<p<\infty$, using Lemma (1.4) (iii) with $\beta=\alpha$ and $\alpha<\beta<2$ respectively.

In case $p=\infty$ and $\Omega=1$, the approximation argument used above fails. However, if $f \in L_{\alpha}^{\infty}$ we can still form (1.6) since $f \in L^{\infty}$ and $\left[|z|^{\alpha} \exp [-\varepsilon|z|]\right]^{\wedge} \in L^{1}$. Moreover since $f=\left(J^{-\alpha} f\right) * G_{\alpha}$, Lemma (1.4) (iii) is valid for $p=\infty$ even though $f \notin C_{0}^{\infty}$, and it follows as usual that $\left\|\tilde{f}_{\varepsilon}(\cdot)-\tilde{f}^{\prime}(\cdot, \varepsilon)\right\|_{\infty} \leqq c\|\dot{f}\|_{\infty, \alpha}$. To show $\left\|\tilde{f}_{\varepsilon}\right\|_{\infty} \leqq c\|f\|_{\infty, \alpha}$ it is therefore enough to show that $\tilde{f}(x, \varepsilon)$ is a constant times the Poisson integral of $J^{-\alpha} f * d \mu \in L^{\infty}$. If $h(z)=h(\varepsilon, z)=|z|^{\alpha} \exp [-\varepsilon|z|]$ then since $f=\left(J^{-\alpha} f\right) * G_{\alpha}$,

$$
\begin{aligned}
\frac{1}{c_{0}^{(\alpha)}} f(x, \varepsilon) & =\int\left[\int\left(J^{-\alpha} f\right)(x+z-y) G_{\alpha}(y) d y\right] \hat{h}(z) d z \\
& =\int\left[\int G_{\alpha}(y+z) \hat{h}(z) d z\right]\left(J^{-\alpha} f\right)(x-y) d y \\
& =\int\left[\int \hat{G}_{\alpha}(z) h(z) \exp [i(y \cdot z)] d z\right]\left(J^{-\alpha} f\right)(x-y) d y .
\end{aligned}
$$

However,

$$
\hat{G}_{\alpha}(z) h(z)=\frac{|z|^{\alpha}}{\left(1+|z|^{2}\right)^{\alpha / 2}} \exp [-\varepsilon|z|]=[\mu(\varepsilon, z)]^{\wedge}
$$

where $\mu(\varepsilon, z)$ denotes the Poisson integral of $d \mu$. Since $\hat{G}_{\alpha} h \in L^{1}$, we obtain by Fourier inversion

$$
f(x, \varepsilon) / c_{0}^{(\alpha)}=\mu(\varepsilon, x) *\left(J^{-\alpha} f\right)(x),
$$

and changing the order of integration we see $f(x, \varepsilon) / c_{0}^{(\alpha)}$ is the Poisson integral of $J^{-\alpha} f * d \mu$.

We conclude $\S 1$ with several remarks.

(1) THEOREM 3. If $f \in L_{\alpha}^{p}, 1<p<\infty, 0<\alpha<2$, and $\Omega$ satisfies the hypothesis of Theorem 1 then

$$
\lim _{\varepsilon \rightarrow 0} f_{\varepsilon}(x)=\lim _{\varepsilon \rightarrow 0}(2 \pi)^{-n} \int_{|z|>\varepsilon}[f(x-z)-f(x)] \frac{\Omega\left(z^{\prime}\right)}{|z|^{n+\alpha}} d z
$$


exists and is finite almost everywhere. The result is also valid for $p=1$ provided $\Omega \equiv 1$.

The proof is similar to that given above and we shall be as brief as possible.

LeMma (1.5). If $f \in L_{\alpha}^{p}$ then for almost every $x$

(i)

$$
\int_{|z|<\varepsilon}|f(x+z)-f(x)| d z=o\left(\varepsilon^{n+\alpha}\right)
$$

if $0<\alpha<1$ and $1<p<\infty$,

$$
\int_{|z|<\varepsilon}\left|f(x+z)-f(x)-\sum_{j=1}^{n} z_{j} \frac{\partial f}{\partial x_{j}}(x)\right| d z=o\left(\varepsilon^{n+\alpha}\right)
$$

if $1 \leqq \alpha<2$ and $1<p<\infty$, and

$$
\int_{|z|<\varepsilon}|f(x+z)+f(x-z)-2 f(x)| d z=o\left(\varepsilon^{n+\alpha}\right)
$$

if $0<\alpha<2$ and $p=1$.

For $\alpha=1$, (ii) is a consequence of Theorem 12 of [3] and the identification of $L_{1}^{p}$ with the classical Sobolev space. If $\alpha$ is not an integer, $0<\alpha<2$ and $f \in L_{\alpha}^{p}$ for $1<p<\infty$, then $J^{-\alpha} f \in L^{p}$ and therefore satisfies

$$
\left(\varepsilon^{-n} \int_{|z|<\varepsilon}\left|\left(J^{-\alpha} f\right)(x+z)-\left(J^{-\alpha} f\right)(x)\right|^{p} d z\right)^{1 / p}=o(1)
$$

almost everywhere. By Theorem 4 of [3], $f=J^{\alpha}\left(J^{-\alpha} f\right)$ satisfies (i) or (ii) almost everywhere. The proof of (iii) follows from the method of the proof of Theorem 4 of [3] and we omit it.

Lemma (1.6). If $f \in L_{\alpha}^{p}, 1 \leqq p<\infty, 0<\alpha<2$, and $\Omega=1$ when $p=1$, then

$$
f_{\varepsilon}(x)-\tilde{f}(x, \varepsilon) \rightarrow 0
$$

as $\varepsilon \rightarrow 0$ at each point where the conclusion of Lemma (1.5) is valid.

Proof. Suppose $0<\alpha<1$ and $1<p<\infty$. Then from Lemmas (1.2) and (1.3)

$$
\begin{aligned}
\left|\tilde{f}_{\varepsilon}(x)-\tilde{f}(x, \varepsilon)\right| \leqq & c_{1} \int_{|z|<\varepsilon}|f(x+z)-f(x)|\left(\frac{\varepsilon}{|z|}\right)^{-n-\alpha}|z|^{-n-\alpha} d z \\
& +c_{2} \int_{|z|>\varepsilon}|f(x+z)-f(x)|\left(\frac{\varepsilon}{|z|}\right)^{1 / 2}|z|^{-n-\alpha} d z .
\end{aligned}
$$

The first integral on the right tends to zero with $\varepsilon$ by (1.7), and the second is majorized by a constant times

$$
\varepsilon^{1 / 2}\left[\int_{\varepsilon<|z|<\delta}+\int_{|z|>\delta}|f(x+z)-f(x)| \frac{d z}{|z|^{n+\alpha+1 / 2}}\right] .
$$


For fixed $\delta>0$,

$$
\varepsilon^{1 / 2} \int_{|z|>\delta}|f(x+z)-f(x)| \frac{d z}{|z|^{n+\alpha+1 / 2}}=O\left(\varepsilon^{1 / 2}\right)
$$

while if $G(t)=\int_{|z|<t}|f(x+z)-f(x)| d z$ then

$$
\begin{aligned}
\varepsilon^{1 / 2} \int_{\varepsilon<|z|<\delta}|f(x+z)-f(x)| \frac{d z}{|z|^{n+\alpha+1 / 2}} & =c \varepsilon^{1 / 2} \int_{\varepsilon}^{\delta} \frac{d G(t)}{t^{n+\alpha+1 / 2}} \\
& =c\left(\left.\varepsilon^{1 / 2} \frac{G(t)}{t^{n+\alpha+1 / 2}}\right|_{\delta} ^{\delta}\right)+c^{\prime} \varepsilon^{1 / 2} \int_{\varepsilon}^{\delta} G(t) \frac{d t}{t^{n+\alpha+3 / 2}} .
\end{aligned}
$$

Since $G(t)=o\left(t^{n+\alpha}\right)$ as $t \rightarrow 0$, the lemma follows in this case. The proof for $1 \leqq \alpha<2$ and $1<p<\infty$ is similar using Lemma (1.5) (ii) and the orthogonality of $\Omega$ to polynomials of degree 1 . When $p=1$ and $\Omega=1$, we use Lemma (1.5) (iii) and argue as above.

To prove Theorem 3, we recall that for $f \in C_{0}^{\infty}, f(x, \varepsilon)$ is the Poisson integral of the sum of a constant times $J^{-\alpha} f * d \mu$ and a singular integral of $J^{-\alpha} f * d \mu$. By Lemmas (1.2) and (1.3) $K_{\varepsilon}(z)$ belongs to all $L^{q}, 1 \leqq q \leqq \infty$, for each $\varepsilon$, and since $C_{0}^{\infty}$ is dense in $L_{\alpha}^{p}, 1 \leqq p<\infty$, it follows $f^{x}(x, \varepsilon)$ is the Poisson integral of an $L^{p}$ function for any $f \in L_{\alpha}^{p}(\Omega=1$ if $p=1)$. Hence $f(x, \varepsilon)$ converges almost everywhere and Theorem 3 follows.

(2) The assumption that $\Omega$ is infinitely differentiable can be considerably relaxed in both Theorem 1 and the preceding remark. This will be the subject of a continuation of this paper to appear in the future.

(3) Finally it was shown in [11] that when $\alpha=1, \tilde{f}(x, \varepsilon)=-\sum_{j=1}^{n} f_{j}(x, \varepsilon)$ when $f_{j}(x, \varepsilon)$ is the Poisson integral of p.v. $(2 \pi)^{-n}\left(\partial f / \partial x_{j} * K_{j}\right), K_{j}(x)=|x|^{-n} x_{j}^{\prime} \Omega\left(x^{\prime}\right)$, $f \in L_{1}^{p}, 1<p<\infty$.

2. In this section we will prove the necessity of Theorem 1. Let us again suppose for the time being that $f \in C_{0}^{\infty}$ and consider (see $\S 1$ )

$$
f_{\varepsilon}^{\wedge}(x)=(2 \pi)^{-n / 2} \hat{f}(x)|x|^{\alpha} \sum_{0}^{\infty}(-i)^{m} w_{m}^{(\alpha)}(\varepsilon|x|) Y_{m}\left(x^{\prime}\right),
$$

which converges pointwise to $\hat{f}(x)|x|^{\alpha} \sum_{0}^{\infty} c_{m}^{(\alpha)} Y_{m}\left(x^{\prime}\right)$. If $f^{x}$ is the limit in $L^{2}$ of $\tilde{f}_{\varepsilon}$ then by the Parseval-Plancherel formula,

$$
\begin{aligned}
\hat{f}^{\wedge}(x) & =\hat{f}(x)|x|^{\alpha} \sum_{0}^{\infty} c_{m}^{(\alpha)} Y_{m}\left(x^{\prime}\right) \\
& =c_{0}^{(\alpha)} Y_{0}\left(x^{\prime}\right) \hat{f}(x)|x|^{\alpha}+\hat{f}(x) \hat{K}(x)|x|^{\alpha},
\end{aligned}
$$

where $R(x)$ is the principal-valued Fourier transform of $K(x)=|x|^{-n} \sum_{1}^{\infty} d_{m}^{(\alpha)} Y_{m}\left(x^{\prime}\right)$.

Suppose $\Omega\left(x^{\prime}\right)=Y_{m}\left(x^{\prime}\right)$ for some $m \geqq 1$. Then

$$
f^{\wedge}(x)=d_{m}^{(\alpha)}|x|^{\alpha} \hat{f}(x)\left[\Omega\left(x^{\prime}\right) /|x|^{n}\right]^{\wedge}
$$


and by Lemma (0.1), if $f^{\prime}=$ p.v. $\left(f * \Omega(x) /|x|^{n}\right)$,

$$
J^{-\alpha} f^{\prime}=\left(1 / d_{m}^{(\alpha)}\right)(\tilde{f} * d \sigma)+(2 \pi)^{-n}\left(f^{\prime} * d \tau\right) .
$$

Taking $L^{p}$ norms, $1<p<\infty$, and observing that $J^{-\alpha} f^{\prime}=\left(J^{-\alpha} f\right)^{\prime}$,

$$
\left\|\left(J^{-\alpha} f\right)^{\prime}\right\|_{p} \leqq d\left(\|f\|_{p}+\|f\|_{p}\right), \quad c=c_{p, \alpha, m} .
$$

Taking successively for $\Omega$ each element $\Omega_{i}, i=1, \ldots, M_{m}$, of a normalized basis for the spherical harmonics of degree $m$ and letting $\tilde{f}(x)$ denote the limit in $L^{p}$ of

$$
(2 \pi)^{-n} \int_{|z|>\varepsilon}[f(x-z)-f(x)] \frac{\Omega_{i}(z)}{|z|^{n+\alpha}} d z,
$$

we obtain (see the introduction)

$$
\|f\|_{p, \alpha}=\left\|J^{-\alpha} f\right\|_{p} \leqq c\left(\sum_{i}\left\|\tilde{f}_{i}\right\|_{p}+\|f\|_{p}\right)
$$

$c=c_{p, \alpha, m}$. Moreover, since $\left\|\tilde{f}_{i}\right\|_{p} \leqq c_{p, \alpha}\|f\|_{p, \alpha}$ for $f \in L_{\alpha}^{p}$ and $C_{0}^{\infty}$ is dense in $L_{\alpha}^{p}, 1<p<\infty$, there is a constant $c=c_{p, \alpha, m}$ such that for any $f \in L_{\alpha}^{p}$

$$
c^{-1}\|f\|_{p, \alpha} \leqq \sum_{i}\left\|\tilde{f}_{i}\right\|_{p}+\|f\|_{p} \leqq c\|f\|_{p, \alpha}
$$

Here we have assumed of course that $m \geqq 1$ when $0<\alpha<1$ and $m \geqq 2$ when $1 \leqq \alpha<2$.

Suppose now that $f \in L^{p}, 1<p<\infty$, and each

$$
(2 \pi)^{-n} \int_{|z|>\varepsilon}[f(x-z)-f(x)] \frac{\Omega_{i}\left(z^{\prime}\right)}{|z|^{n+\alpha}} d z=(2 \pi)^{-n} \int_{|z|>\varepsilon} f(x-z) \frac{\Omega_{i}\left(z^{\prime}\right)}{|z|^{n+\alpha}} d z
$$

converges in $L^{p}$ to a limit $f_{i}$, where $\left\{\Omega_{i}\right\}$ is a normalized basis for the spherical harmonics of a fixed degree $m \geqq 1$. Pick $\phi \in \mathscr{S}$ (the Schwartz space of rapidly decreasing functions) with $\phi \geqq 0$ and $\int \phi(x) d x=1$ and set $\phi_{\delta}(x)=\delta^{-n} \phi(x / \delta), \delta>0$, $f(x, \delta)=\left(f * \phi_{\delta}\right)(x)$. Then

$$
\begin{aligned}
\int_{|z|>\varepsilon} f(x-z, \delta) \frac{\Omega_{i}(z)}{|z|^{n+\alpha}} d z & =\int_{|z|>\varepsilon}\left[\int_{E^{n}} f(x-z-y) \phi_{\delta}(y) d y\right] \frac{\Omega_{i}(z)}{|z|^{n+\alpha}} d z \\
& =\int_{E^{n}}\left[\int_{|z|>\varepsilon} f(x-y-z) \frac{\Omega_{i}(z)}{|z|^{n+\alpha}} d z\right] \phi_{\delta}(y) d y
\end{aligned}
$$

converges in $L^{p}$ as $\varepsilon \rightarrow 0$ to $\int_{E^{n}} f_{i}(x-y) \phi_{\delta}(y) d y$, whose $L^{p}$ norm is bounded by $\left\|\tilde{f}_{i}\right\|_{p}\|\phi\|_{1}=\left\|\tilde{f}_{i}\right\|_{p}$ for all $\delta>0$. Since $f(x, \delta) \in L_{\alpha}^{p}$ for each fixed $\delta>0$, it follows from (2.3) that

$$
\left\|J^{-\alpha} f(x, \delta)\right\|_{p} \leqq c\left(\sum\left\|\tilde{f}_{i}\right\|_{p}+\|f\|_{p}\right) .
$$

Pick $\delta_{k} \rightarrow 0$ so that $J^{-\alpha} f\left(x, \delta_{k}\right)$ converges weakly to $h(x) \in L^{p}$. Then $J^{-\alpha} f\left(x, \delta_{k}\right)$ converges to $h(x)$ in the sense of distributions. If $g=J{ }^{-\alpha} f, g \in \mathscr{S}^{\prime}$ (the space of tempered distributions), then

$$
\left[J^{-\alpha} f\left(x, \delta_{k}\right)\right]^{\wedge}=\hat{f}(x)\left(1+|x|^{2}\right)^{\alpha / 2}\left[\phi_{\delta_{k}}(x)\right]^{\wedge}=\hat{g} \hat{\phi}\left(\delta_{k} x\right) .
$$


Since $\hat{\phi}\left(\delta_{k} x\right)-\hat{\phi}(0)=\hat{\phi}\left(\delta_{k} x\right)-1$ and all its derivatives are bounded and converge uniformly to zero on compact sets, $\left[J^{-\alpha} f\left(x, \delta_{k}\right)\right]^{\wedge} \rightarrow \hat{g}$ in the sense of distributions. Hence $J^{-\alpha} f\left(x, \delta_{k}\right) \rightarrow g$ as distributions, and therefore the action of $g$ on a function $\psi \in \mathscr{S}$ is given by $\int_{E^{n}} h(x) \psi(x) d x$. Since $h \in L^{p}, f \in L_{\alpha}^{p}$ and the theorem is proved in this case.

The case $\Omega=1$ and $0<\alpha<2$ is practically the same. For if $f \in C_{0}^{\infty}$ and $\tilde{f}(x)$ is the limit of

$$
f_{\varepsilon}(x)=(2 \pi)^{-n} \int_{|z|>\varepsilon}[f(x-z)-f(x)] \frac{d z}{|z|^{n+\alpha}},
$$

then $\tilde{f}^{\wedge}(x)=c_{0}^{(\alpha)} \hat{f}(x)|x|^{\alpha}$ and the analogue of $(2.2)$ is

$$
J^{-\alpha} f=a(\tilde{f} * d \sigma)+b(f * d \tau)
$$

where $a$ and $b$ depend only on $\alpha$ and $n$. Hence for $f \in L_{\alpha}^{p}, 1<p<\infty$,

$$
c^{-1}\|f\|_{p, \alpha} \leqq\|f\|_{p}+\|f\|_{p} \leqq c\|f\|_{p, \alpha} .
$$

The remainder is similar to the proof above and we obtain that $f \in L_{\alpha}^{p}$ if $f \in L^{p}$ and $\tilde{f_{\varepsilon}}$ converges in $L^{p}, 1<p<\infty$.

Again the results have analogues for $p=1$ and $p=\infty$ provided we take $\Omega=1$. In fact for $p=1$ and $\Omega=1$ the argument just given is still valid, if we use (2.4) for $f(x, \delta)$ to show $J^{-\alpha} f(x, \delta)$ converges in $L^{1}$. For $p=\infty$ and $\Omega=1$, however, it must be modified.

Let $f \in L_{\alpha}^{\infty}$ so that $\left\|\tilde{f}_{\epsilon}\right\|_{\infty} \leqq C$,

$$
f_{\varepsilon}(x)=(2 \pi)^{-n} \int_{|z|>\varepsilon}[f(x-z)-f(x)] \frac{d z}{|z|^{n+\alpha}} .
$$

Choose $\varepsilon_{k} \rightarrow 0$ so that $\tilde{f}_{\varepsilon_{k}}$ converges in the weak-star topology of $L^{\infty}$ to a function $\tilde{f}$, $\|f\|_{\infty} \leqq C$. We claim there are constants $a$ and $b$ depending only on $\alpha$ and $n$ such that $J^{-\alpha} f=a\left(f^{\tilde{*}} * d \sigma\right)+b(f * d \tau)$ almost everywhere. If $l \in \mathscr{S}^{\prime}$ and $\psi \in \mathscr{S}$, let $\langle l, \psi\rangle$ denote the action of $l$ on $\psi$; and for any $g$, let $g_{1}(x)=g(-x)$. Then

$$
\begin{aligned}
\int_{E^{n}}\left(J^{-\alpha} f\right) \psi d x & =\left\langle J^{-\alpha} f, \psi\right\rangle=\left\langle\hat{f},\left(1+|x|^{2}\right)^{\alpha / 2} \hat{\psi}_{1}\right\rangle \\
& =\left\langle f,\left[\left(1+|x|^{2}\right)^{\alpha / 2} \hat{\psi}_{1}\right]^{\wedge}\right\rangle \\
& =\left\langle f, a\left(\tilde{\psi}_{1} * d \sigma\right)_{1}+b\left(\psi_{1} * d \tau\right)_{1}\right\rangle \\
& =a \int_{E^{n}} f\left(\tilde{\psi}_{1} * d \sigma\right)_{1} d x+b \int_{E^{n}} f\left(\psi_{1} * d \tau\right)_{1} d x .
\end{aligned}
$$

Since $\int_{E^{n}} f\left(\psi_{1} * d \tau\right)_{1} d x=\int_{E^{n}}(f * d \tau) \psi d x$, our claim will follow if we show

$$
\int_{E^{n}} f\left(\tilde{\psi}_{1} * d \sigma\right)_{1} d x=\int_{E^{n}}(\tilde{f} * d \sigma) \psi d x .
$$


Taking Fourier transforms it is easy to see that $\tilde{\psi}_{1}=(\tilde{\psi})_{1}$ and $\tilde{\psi}(x+y)=[\psi(x+y)]^{\sim}$. Moreover, $\int_{E^{n}} \tilde{f}_{\varepsilon_{k}} \psi d x=\int_{E^{n}} f \tilde{\psi}_{\varepsilon_{k}} d x, \int_{E^{n}} \tilde{f}_{\varepsilon_{k}} \psi d x \rightarrow \int_{E^{n}} \tilde{f} \psi d x$ by the definition of $\tilde{f}$, and $\int_{E^{n}} f \tilde{\psi}_{\varepsilon_{k}} d x \rightarrow \int_{E^{n}} f \tilde{\psi} d x$ since $\tilde{\psi}_{\varepsilon_{k}}$ converges to $\tilde{\psi}$ in $L^{1}$ (cf. Theorem 1 with $p=1, \Omega=1)$ and $f \in L^{\infty}$. Hence $\int_{E^{n}} \tilde{f} \psi d x=\int_{E^{n}} f \tilde{\psi} d x$ and

$$
\begin{aligned}
\int_{E^{n}} f\left(\tilde{\psi}_{1} * d \sigma\right)_{1} d x & =\int_{E^{n}} d \sigma(y) \int_{E^{n}} f(x) \tilde{\psi}_{1}(-x-y) d x \\
& =\int_{E^{n}} d \sigma(y) \int_{E^{n}} f(x) \tilde{\psi}(x+y) d x \\
& =\int_{E^{n}} d \sigma(y) \int_{E^{n}} f(x)[\psi(x+y)]^{\sim} d x \\
& =\int_{E^{n}} d \sigma(y) \int_{E^{n}} \tilde{f}(x) \psi(x+y) d x \\
& =\int_{E^{n}} \psi(x) d x \int_{E^{n}} f(x-y) d \sigma(y),
\end{aligned}
$$

which establishes the claim. Taking $L^{\infty}$ norms, it follows $\|f\|_{\infty, \alpha} \leqq c^{\prime}\left(C+\|f\|_{\infty}\right)$ for $f \in L_{\alpha}^{\infty}$ with $\left\|f_{\varepsilon}\right\|_{\infty} \leqq C$.

Suppose now that $f \in L^{\infty}$ and $\left\|\tilde{f}_{\varepsilon}\right\|_{\infty} \leqq C$. Let $f(x, \delta)=\left(f * \phi_{\delta}\right)(x)$ where $\phi_{\delta}$ is the approximation to the identity introduced earlier. Then $\|f(x, \delta)\|_{\infty} \leqq\|f\|_{\infty}$, $\left\|(f(x, \delta))_{\varepsilon}\right\|_{\infty}=\left\|\left(f_{\varepsilon}^{*} * \phi_{\delta}\right)\right\|_{\infty} \leqq\left\|f_{\varepsilon}^{f}\right\|_{\infty} \leqq C$, and $f(x, \delta) \in L_{\alpha}^{\infty}$. By the above,

$$
\left\|J^{-\alpha} f(x, \delta)\right\|_{\infty} \leqq c^{\prime}\left(C+\|f\|_{\infty}\right),
$$

and we can pick $\delta_{k}$ such that $J^{-\alpha} f\left(x, \delta_{k}\right)$ converges in the weak-star topology to $h(x) \in L^{\infty}$. It now follows as usual that $f \in L_{\alpha}^{\infty}$.

REMARK. We add one final remark concerning the $\Lambda(\alpha, p, q)$ spaces. For the definition and properties of these spaces, we refer the reader to [8] and [9].

If $f \in L_{\alpha}^{p} \cap \Lambda(\alpha, p, q)$ where $1 \leqq p<\infty, 1 \leqq q \leqq \infty$, and $0<\alpha<2$ then $f \in \Lambda(0, p, q)$ and $\|\tilde{f}\|_{\Lambda(0, p, q)} \leqq c\|f\|_{\Lambda(\alpha, p, q)}$ with $c$ independent of $f$. Here of course $\Omega \equiv 1$ if $p=1$. Moreover, if $f \in \Lambda(0, p, q) \cap L_{\alpha}^{p}$ and each

$$
f_{i}(x)=\lim _{\varepsilon \rightarrow 0} \int_{|z|>\varepsilon}[f(x-z)-f(x)] \frac{\Omega_{i}(z)}{|z|^{n+\alpha}} d z
$$

belongs to $\Lambda(0, p, q)$ where $\left\{\Omega_{i}\right\}$ is a normalized basis for the spherical harmonics of degree $m(m \neq 1$ if $1 \leqq \alpha<2$ and $m=0$ if $p=1)$ then $f \in \Lambda(\alpha, p, q)$.

In view of the inclusion relations between $L_{\alpha}^{p}$ and $\Lambda(\alpha, p, q)$ (see [8, p. 452]), this remark is trivial in many cases.

If $f \in C_{0}^{\infty}$, the formula in section 2 for $f^{\wedge}$ and Lemma (0.1) imply that the Poisson integral of $f$ is the Poisson integral of the sum of a constant times $J^{-\alpha} f * d \mu$ and a singular integral of $J^{-\alpha} f * d \mu$. Hence the same is true for any $f \in L_{\alpha}^{p}$ and

$$
\left\|f^{f}\right\|_{\Lambda(0, p, q)} \leqq c\left\|J^{-\alpha} f\right\|_{\Lambda(0, p, q)}=c\|f\|_{\Lambda(\alpha, p, q)}
$$

(see $[9$, p. 827-829]). 
Conversely, if $f \in L_{\alpha}^{p}$ it is easy to see that (2.2) (or (2.4)) holds. Hence if, for example, $1<p<\infty$, we obtain by taking the $\Lambda(0, p, q)$ norm

$$
\|f\|_{\Lambda(\alpha, p, q)} \leqq c\left(\|f\|_{\Lambda(0, p, q)}+\sum_{i}\left\|\tilde{f}_{i}\right\|_{\Lambda(0, p, q)}\right)
$$

for $f \in \Lambda(\alpha, p, q)$. For any $f \in \Lambda(0, p, q) \cap L_{\alpha}^{p}$ we apply the last inequality to the Poisson integral of $f$ and invoke [8, Lemma 5, p. 426].

\section{REFERENCES}

1. N. Aronszajn and K. T. Smith, Theory of Bessel potentials. I, Ann. Inst. Fourier (Grenoble) 11 (1961), 385-475.

2. A. P. Calderón, Lebesgue spaces of functions and distributions, Proc. Sympos. Pure Math., Vol 4, Amer. Math. Soc., Providence, R. I., 1967, pp. 33-49.

3. A. P. Calderón and A. Zygmund, Local properties of solutions of elliptic partial differential equations, Studia Math. 20 (1961), 171-225.

4. —_ On the existence of certain singular integrals, Acta Math. 88 (1952), 85-139.

5. A. Erdélyi, et al., Higher transcendental functions, Vol. 1, McGraw-Hill, New York, 1953.

6. _- Vol. 2, McGraw-Hill, New York, 1953.

7. E. M. Stein, The characterization of functions arising as potentials, Bull. Amer. Math. Soc. 67 (1961), 102-104.

8. M. H. Taibleson, On the theory of Lipschitz spaces of distributions on Euclidean n-space. I. Principal properties, J. Math. Mech. 13 (1964), 407-479.

9. - On the theory of Lipschitz spaces of distributions on Euclidean n-space. II. Translation invariant operators, duality and interpolation, J. Math. Mech. 14 (1965), 821-840.

10. G. N. Watson, A treatise on the theory of Bessel functions, Cambridge Univ. Press, Cambridge, 1962.

11. R. L. Wheeden, Hypersingular integrals and summability of Fourier integrals and series, Proc. Sympos. Pure Math., Vol. 10, Amer. Math. Soc., Providence, R. I., 1967, pp. 336-369.

INSTITUTE FOR ADVANCED STUDY,

Princeton, NeW Jersey 